Research Article

\title{
Combination Therapy with Disulfiram, Copper, and Doxorubicin for Osteosarcoma: In Vitro Support for a Novel Drug Repurposing Strategy
}

\author{
Jonathan B. Mandell, ${ }^{1,2}$ Feiqi Lu,, ${ }^{1,3}$ Matthew Fisch, ${ }^{1}$ Jan H. Beumer, ${ }^{4,5}$ Jianxia Guo, ${ }^{5}$ \\ Rebecca J. Watters, ${ }^{1,5,6}$ and Kurt R. Weiss $\mathbb{D}^{1,7}$ \\ ${ }^{1}$ Musculoskeletal Oncology Laboratory, Department of Orthopaedic Surgery, University of Pittsburgh, Pittsburgh, PA, USA \\ ${ }^{2}$ Department of Infectious Diseases and Microbiology, University of Pittsburgh, Pittsburgh, PA, USA \\ ${ }^{3}$ School of Medicine, Tsinghua University, Beijing, China \\ ${ }^{4}$ Department of Pharmaceutical Sciences, University of Pittsburgh, Pittsburgh, PA, USA \\ ${ }^{5}$ UPMC Hillman Cancer Center, Pittsburgh, PA, USA \\ ${ }^{6}$ Department of Pharmacology and Chemical Biology, University of Pittsburgh, Pittsburgh, PA, USA \\ ${ }^{7}$ Departments of Anatomic Pathology and General Surgical Oncology, University of Pittsburgh, Pittsburgh, PA, USA
}

Correspondence should be addressed to Kurt R. Weiss; weiskr@upmc.edu

Received 6 April 2019; Accepted 19 May 2019; Published 11 July 2019

Academic Editor: Dae-Geun Jeon

Copyright (C) 2019 Jonathan B. Mandell et al. This is an open access article distributed under the Creative Commons Attribution License, which permits unrestricted use, distribution, and reproduction in any medium, provided the original work is properly cited.

\begin{abstract}
Although many cancer cells have significantly higher copper concentrations compared with normal cells and tissues, the role of copper in cancer biology and metastatic disease remains poorly understood. Here, we study the importance of copper in osteosarcoma, which frequently metastasizes to the lungs and is often chemoresistant. K12 and K7M2 are murine OS cells with differing metastatic phenotypes: K7M2 is highly metastatic, whereas K12 is much less so. Intracellular copper levels were determined using atomic absorption. Copper transporters were quantified by qPCR. Cytotoxicity of doxorubicin, disulfiram, and copper(II) chloride was assessed with a cell viability fluorescence stain. Additionally, K7M2 viable cell counts were determined by trypan blue exclusion staining after 72 hours of treatment. Copper levels were found to be significantly higher in K12 OS cells than in K7M2 cells. qPCR showed that K12 cells upregulate the copper influx pump CTR1 and downregulate the copper efflux pump ATP7A compared to K7M2 OS cells. Combination treatment of copper chloride (50 nM) with disulfiram (80 nM) was only cytotoxic to K12 cells. Triple treatment with doxorubicin, disulfiram, and copper displayed potent and durable cytotoxicity of highly metastatic K7M2 cells. We demonstrate here that murine OS cell lines differing in metastatic potential also vary in endogenous copper levels and regulation. Additionally, these differences in copper regulation may contribute to selective cytotoxicity of K12 cells by extremely low doses of copper-potentiated disulfiram. The combination of doxorubicin, disulfiram, and copper should be explored as a therapeutic strategy against OS metastases.
\end{abstract}

\section{Introduction}

Osteosarcoma (OS) is the most common primary malignancy of the bone and mainly afflicts children and teens. Virtually, all metastatic disease is to the lungs, which ultimately causes patient mortality. Before the chemotherapeutic era, 5-year survival with surgery alone was only 10-20\%. Modern treatment paradigms employ neoadjuvant (preoperative) chemotherapy, surgery, and adjuvant (postoperative) chemotherapy. The combination of cytotoxic chemotherapy and surgical ablation has improved 5-year survival to approximately $65 \%$ in most large series. Although several chemotherapeutic agents are used to treat OS, doxorubicin (Dox) has functioned as the backbone of OS chemotherapy for decades. Dox is considered to be an indispensable component for worldwide treatment of OS [1-12]. 
The addition of Dox to the treatment OS has dramatically improved outcomes, but enthusiasm is limited by several realities. Most importantly, the prognoses of children with OS have not improved in over three decades despite multiple clinical trials [13-17]. Children who present with pulmonary metastases at the time of diagnosis, or develop them during the course of their treatment, have especially poor prognoses of 15-30\% 5-year survival [6]. Additionally, children who survive OS do so at great cost. Dox is extraordinarily cardiotoxic, causing dose-dependent cardiomyopathy that leads to congestive heart failure and death. Two recent reviews of long-term pediatric sarcoma survivors found incidences of severe cardiotoxicity of $26 \%$ and $28 \%$ $[18,19]$. Therefore, the two greatest obstacles that prevent improvement of the prognoses of OS patients are: (1) the absence of treatments that specifically target OS metastatic biology, and (2) the deleterious long-term consequences of Dox treatment for OS survivors. The efficacy of conventional cytotoxic chemotherapy regimens in OS have likely reached their zenith, necessitating the application of more biologically intelligent approaches.

K7M2 and K12 are murine OS cell lines that are both derived from the same parental tumor in Balb/cJ mouse. K7M2 is aggressively metastatic to the lung, whereas K12 displays a weakly metastatic phenotype. As these cell lines are clonally related but display dramatically different metastatic abilities, they are a simple yet powerful tool to study OS metastatic biology [20, 21]. Previous work from our group utilized K7M2 and K12 to identify and investigate factors that confer metastatic potential to OS cells [22-25]. K7M2 cells express and produce significantly greater amounts of the cancer stem cell marker aldehyde dehydrogenase $(\mathrm{ALDH})$ compared with K12 cells, suggesting that ALDH is a metastasis-associated factor in OS $[23,24,26]$. ALDH enables OS cells to withstand oxidative stress, and OS cells with high ALDH expression are more motile and invasive than OS cells with lower ALDH expression. These findings led us to investigate the ability of the ALDH inhibitor, disulfiram (Dis), to alter the metastatic phenotypes of OS cells. We observed that Dis renders OS cells more susceptible to oxidative stress, alters OS cell morphology, and diminishes their viability and invasiveness. We also demonstrated dose-dependent cytotoxicity of Dis in the cultured cells of bone sarcoma patients from our clinic [27].

Copper $(\mathrm{Cu})$ is an essential micronutrient for physiologic redox reactions and also important to the oncogenic processes of invasion and metastasis [28, 29]. Many neoplasia conditions display higher levels of endogenous intratumoral $\mathrm{Cu}$ compared with normal cells and tissues $[30,31]$. Patients with more aggressive and chemotherapyresistant diseases have demonstrated elevated serum $\mathrm{Cu}$ levels $[30,31]$. Several basic and clinical studies have suggested that Dis's antitumor activity is enhanced by the addition of $\mathrm{Cu}$ (II) compounds [28, 32-37]. However, evaluation of the differences in $\mathrm{Cu}$ metabolism between low grade vs. highly metastatic tumors and how this directly relates to the success of $\mathrm{Dis}+\mathrm{Cu}(\mathrm{II})$ treatment have not been explored.
In this report, we investigated differences in $\mathrm{Cu}$ metabolism and $\mathrm{Dis}+\mathrm{CuCl}_{2}$ treatment between $\mathrm{K} 12$ and $\mathrm{K} 7 \mathrm{M} 2$ cells. Intracellular $\mathrm{Cu}$ levels of K7M2 and K12 cells with and without treatments were also measured. We also determined expression levels of genes related to both $\mathrm{Cu}$ transport and chemotherapy resistance. Finally, we tested the ability of $\mathrm{CuCl}_{2}$ to alter the potency of Dis treatment in murine OS cells, as well as the potential of Dis $+\mathrm{CuCl}_{2}$ to reduce the cytotoxic dose of Dox. We hypothesized that OS cells with differing metastatic potentials possess varied endogenous $\mathrm{Cu}$ levels and altered gene expression patterns relating to $\mathrm{Cu}$ metabolism and drug resistance. We suspected that $\mathrm{Cu}$ would alter susceptibility of OS cells to Dis-mediated toxicity, and that the combination of $\mathrm{Dis}+\mathrm{CuCl}_{2}$ might permit cytotoxicity with lower Dox doses.

\section{Materials and Methods}

2.1. Cell Lines and Cell Culturing. The highly metastatic OS K7M2 cell line was purchased from ATCC, and K12 cells were generously provided by Dr. Chand Khanna in the Pediatric Oncology Branch of the National Cancer Institute. Both cell lines were sent to IDEXX BioResearch labs to test for the presence of pathogens and mycoplasma, interspecies contamination, and STR profiling to definitively prove a positive identity of the clonally related OS cell lines. Both cell lines were grown and maintained using identical culture media and conditions. Cells were grown in T75 flasks (Corning) with culture media of DMEM (Corning) supplemented with 10\% FBS (Gibco) and 1\% penicillin/ streptomycin $\mathrm{Ab}$ solution (Gibco) at $37^{\circ} \mathrm{C}$ in a humidified incubator.

2.2. Atomic Absorption (AA) Spectrophotometric Cu Analysis. At $\sim 80 \%$ confluency, both cell lines were washed with sterile Dulbecco's phosphate buffered saline (dPBS) and treated with TrypLE (Gibco) for 8 minutes at $37^{\circ} \mathrm{C}$. Cells were given FBS (Gibco) containing culture media and centrifuged at $1500 \mathrm{rpm}$ for 6 minutes. Cell pellets were washed with ice cold, sterile dPBS (Gibco), and cell counts were obtained using an automated cell counter (Bio-Rad). Cells were washed again with ice cold dPBS, centrifuged, and dry cell pellets were stored at $-80^{\circ} \mathrm{C}$. Additionally, K7M2 and $\mathrm{K} 12$ were treated over 24 hours with Dis $(3 \mu \mathrm{M}), \mathrm{CuCl}_{2}(1 \mu \mathrm{M})$, and $\mathrm{Dis}+\mathrm{CuCl}_{2}(0.1$ and $0.25 \mu \mathrm{M})$ then processed in the identical manner as untreated cells.

Copper concentrations were determined using a Perkin Elmer AAnalyst 600 atomic absorption spectrophotometer adjusted to detect $\mathrm{Cu}(324.8 \mathrm{~nm})$. The AAS was operated as a flameless furnace system. Calibration samples were prepared by diluting the copper stock solution with $1 \% \mathrm{HNO}_{3}$ to generate concentrations of 20,50, 100, 200, 500, and $1000 \mathrm{ng} / \mathrm{mL}$. Quality control samples were prepared at 60, 400 , and $800 \mathrm{ng} / \mathrm{mL}$.

Modifier solution consisted of $0.1 \% \mathrm{Pd}$ and $0.06 \%$ $\mathrm{Mg}\left(\mathrm{NO}_{3}\right)_{2}$ in water, which was mixed $1: 1$ (v/v) with sample before injecting $10 \mu \mathrm{L}$. Temperature programming was as per instruction manual. Cell pellets (minimum $2 \cdot 10^{5}$ cells) 
were lysed with $35 \mu \mathrm{L} 0.25 \%$ triton. An aliquot of $5 \mu \mathrm{L}$ was taken for determination of protein using the Bio-Rad Protein assay following the manufacturer's instructions with bovine serum albumin as the standard. A further $30 \mu \mathrm{L}$ of cell lysate was incubated with $30 \mu \mathrm{L}$ of $63 \% \mathrm{HNO}_{3}$ overnight at $60^{\circ} \mathrm{C}$. Next, the sample was diluted with $40 \mu \mathrm{l}$ of $0.2 \% \mathrm{HNO}_{3}$ and next diluted with modifier. Any other dilutions of samples (prior to addition of modifier) were done with $0.2 \% \mathrm{HNO}_{3}$. The final concentration of $\mathrm{Cu}$ in media samples was calculated as total $\mu \mathrm{g} / \mathrm{ml}$. Intracellular $\mathrm{Cu}$ concentration was calculated as: (final sample concentration of $\mathrm{Cu}(\mathrm{ng} / \mathrm{mL})) /($ total sample protein concentration $(\mathrm{mg} /$ $\mathrm{ml})$ ) and recorded as ng.Cu/mg Protein.

2.3. Quantitative PCR. mRNA was collected from $\mathrm{K} 12$ and K7M2 using RNeasy Kit (Qiagen), and cDNA was obtained using Reverse Transcriptase Kit (Applied Biosystems). qPCR was performed using SYBR Green Supermix (BioRad). NoNo, Rps17, and Rps18 reference genes were selected from our previous work [38] for the most stable expression across multiple murine cell and tissue types. Delta CT values were derived from the geometric mean of the three selected housekeeper genes. Differential gene expression between $\mathrm{K} 12$ and K7M2 was determined for $\mathrm{Cu}$ influx pump (CTR1) and $\mathrm{Cu}$ efflux pump (ATP7A), aldehyde dehydrogenase $(\mathrm{ALDH})$, and P-glycoprotein (ABCB1).

2.4. Cytotoxicity Assays. $\mathrm{K} 12$ and $\mathrm{K} 7 \mathrm{M} 2$ were plated in black-walled 96 -well plates $(5,000$ and 10,000 cells, respectively) and cultured for 24 hours. Media was replaced with culture media with fold dilutions of Dox, $\mathrm{CuCl}_{2}$, and Dis $(1 \mathrm{nM}-10 \mu \mathrm{M})$. Additionally, Dis fold dilutions were tested in combination with fixed concentrations of $\mathrm{CuCl}_{2}$ (50, 200, and $800 \mathrm{nM}) .24$ hours after drug addition, cells were stained with a mammalian LIVE/DEAD cell viability kit (Invitrogen). Microscopy of cells was performed with a Nikon ECLIPSE TE2000 U using Northern Eclipse software. Live signal (\%) reduction from the untreated controls was determined using FIJI image mean fluorescence intensity.

2.5. K7M2 Cytotoxicity and Recovery Assays. K7M2 cells were plated $\left(0.1 \times 10^{6}\right)$ in 6-well plates and cultured for 24 hours. Cells were treated over 72 hours with $\mathrm{CuCl}_{2}$ $(0.12-2 \mu \mathrm{M})$, Dis $(0.5-4 \mu \mathrm{M})$, Dox $(0.02-1.2,10 \mu \mathrm{M})$, and Dis $(0.12-2 \mu \mathrm{M})+\mathrm{CuCl}_{2}(0.12-0.25 \mu \mathrm{M})$. Cells were washed with dPBS, treated with $100 \mu \mathrm{L}$ TrypLE Express, and resuspended in culture media. Viable cell counts were obtained using a Trypan Blue exclusion assay and a hemocytometer. Treated K7M2 were recultured with fresh media without drugs in 6-well plates and monitored for robust recovery of cells. Additionally, K7M2 were treated with sublethal doses of Dox $(100 \mathrm{nM})$, Dis $(100 \mathrm{nM})$, and $\mathrm{CuCl}_{2}$ $(250 \mathrm{nM})$ alone, in combination treatments, and triple combination treatments.

2.6. Statistics. All statistical methods were performed using Prism 7.0 (GraphPad, La Jolla CA). Multiple groups were compared using an ordinary one-way ANOVA. Two groups were compared using an unpaired $t$-test. In all cases, $p<0.05, p<0.005, p<0.0005$, and $p<0.0001$ were considered significant, as indicated in each figure.

\section{Results}

3.1. Less Metastatic K12 OS Cells Display Higher Endogenous and Therapeutic Levels of Intracellular Cu Compared to Highly Metastatic K7M2. We first assessed differences in endogenous intracellular $\mathrm{Cu}$ levels between clonally related murine OS cell lines differing in metastatic potential using atomic absorption (AA) spectrophotometry. Intracellular Cu levels were found to be significantly higher in low-metastatic K12 OS cells than in highly metastatic K7M2 (Figure 1(a)). Atomic absorption spectrophotometry was also performed on the FBS lot used for all experiments, DMEM, culture media (DMEM with 10\% FBS), and culture media containing Dis $(3 \mu \mathrm{M}), \mathrm{CuCl}_{2}(1 \mu \mathrm{M})$, and $\mathrm{Dis}+\mathrm{CuCl}_{2}$ $(0.1+0.25 \mu \mathrm{M})$ (Figure 1(b)). Not surprisingly, the main $\mathrm{Cu}$ source was identified as FBS in our cell culture. Both K12 and K7M2 were treated with Dis, $\mathrm{CuCl}_{2}$, and $\mathrm{Dis}+\mathrm{CuCl}_{2}$ over 24 hours. Intracellular $\mathrm{Cu}$ concentrations of $\mathrm{K} 12$ were significantly higher than those of K7M2 after treatment with $1 \mu \mathrm{M} \mathrm{CuCl}_{2}$ and $100 \mathrm{nM}$ Dis $+250 \mathrm{nM} \mathrm{CuCl}_{2}$ (Figure $1(\mathrm{c})$ ). Dis $+\mathrm{CuCl}_{2}$-treated $\mathrm{K} 12$ cells displayed significantly increased intracellular $\mathrm{Cu}$ levels compared to all other $\mathrm{K} 12$ treated groups. Dis $+\mathrm{CuCl}_{2}$ treated K7M2 did not display significantly increased $\mathrm{Cu}$ levels compared to all other K7M2 treated groups (Figure 1(c)).

\subsection{Significantly Altered Expression Levels in Copper Pumps} and Chemoresistance Factors Are Observed in K12 and K7M2 Cells. Due to significant differences seen in endogenous intracellular $\mathrm{Cu}$ levels between $\mathrm{K} 12$ and $\mathrm{K} 7 \mathrm{M} 2$, we next performed quantitative PCR to compare expression-level differences of genes important for $\mathrm{Cu}$ regulation and function in both OS cell lines. qPCR showed that highly metastatic K7M2 cells had significantly lower transcript levels of the $\mathrm{Cu}$ influx pump CTR1 compared with K12 (Figure 2(a)). Conversely, K7M2 showed significantly higher transcript levels of the copper efflux pump ATP7A compared with K12 (Figure 2(b)). Additionally, we tested transcript levels of important metastatic factors. In line with our previous findings [25], qPCR displayed significantly higher expression of aldehyde dehydrogenase (ALDH) in K7M2 compared to K12 (Figure 2(c)). Interestingly, K7M2 also displayed significantly higher expression of the ATPdependent multidrug pump P-glycoprotein (ABCB1) compared with K12 (Figure 2(d)).

\section{3. $\mathrm{CuCl}_{2}$ Potentiation of Disulfiram Results in Significant} Selective Cytotoxicity to K12 Cells and Less to K7M2. Given the significant and dramatic upregulation of ALDH expression in K7M2, as well as the retention of intracellular $\mathrm{Cu}$ levels in $\mathrm{K} 12$, we tested Cu-potentiated Dis cytotoxicity in both OS cell lines over 24 hours. The commonly administered anthracycline chemotherapy drug, Dox, showed 

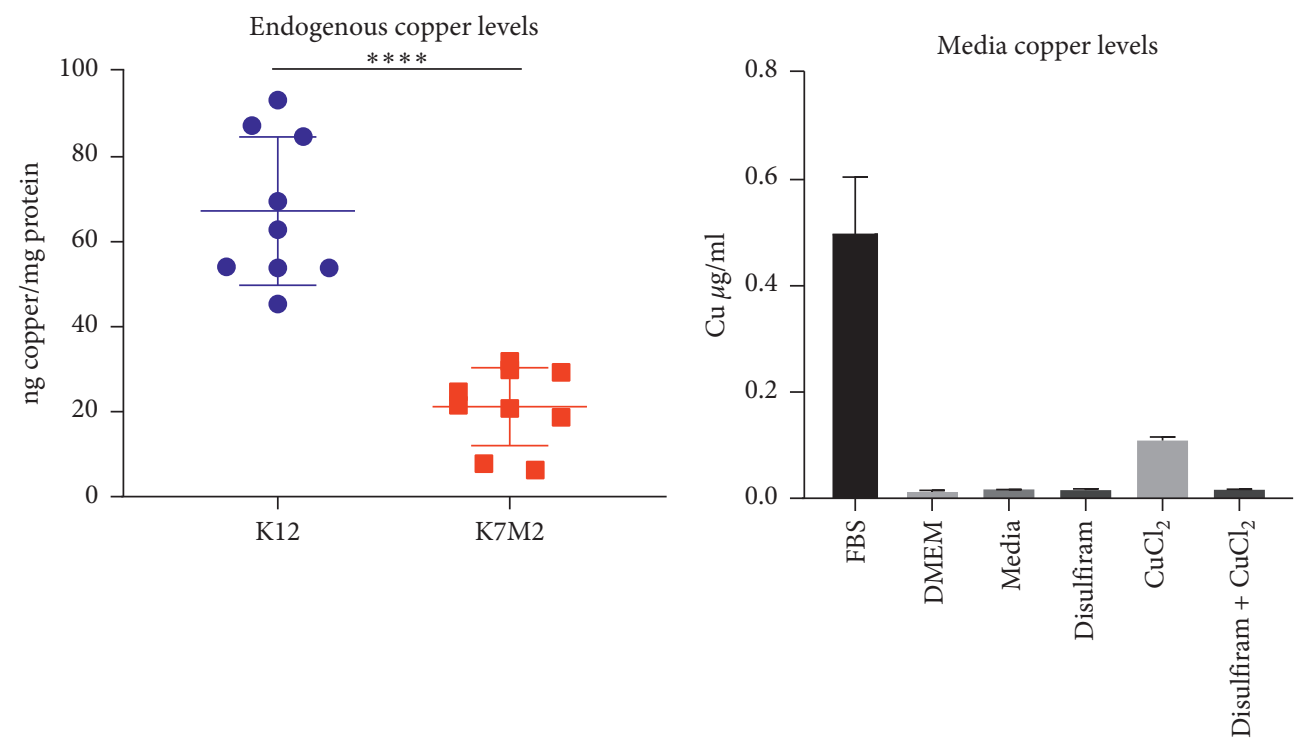

(a)

(b)

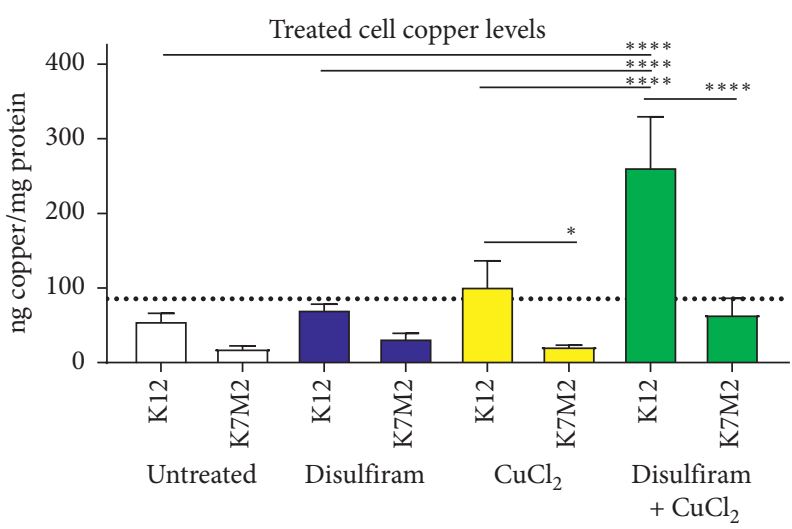

(c)

FIgURE 1: Murine OS cell line K12 displays higher levels of intracellular Cu compared to K7M2. Clonally related OS cell lines (K12 and K7M2) were cultured under identical conditions and then collected for protein quantification and intracellular Cu analysis using atomic absorption (AA) spectrophotometry. (a) Low metastatic K12 cells displayed significantly higher intracellular Cu levels than K7M2 ( $n=6)$. (b) AA analysis of media components and media containing Dis $(3 \mu \mathrm{M}), \mathrm{CuCl}_{2}(1 \mu \mathrm{M})$, and $\mathrm{Dis}+\mathrm{CuCl}_{2}(0.1$ and $0.25 \mu \mathrm{M}$, respectively) was performed $(n=3)$. (c) Both K12 and K7M2 cells were cultured or media treated with Dis, $\mathrm{CuCl}_{2}$, or Dis $+\mathrm{CuCl}_{2}$ at doses previously AA tested $(n=3)$. Copper analysis was performed using three independently cultured and processed samples. ${ }^{*} p<0.05 ;{ }^{* * * *} p<0.0001$.

equivalent cytotoxicity at high doses for $\mathrm{K} 12$ at $\mathrm{IC}_{50} 4.5 \mu \mathrm{M}$ and $\mathrm{K}_{7 \mathrm{M} 2}$ at $\mathrm{IC}_{50} 5.2 \mu \mathrm{M}$ (Figure $3(\mathrm{a})$ ). $\mathrm{CuCl}_{2}$ was not significantly cytotoxic in our LIVE/DEAD assays after 24 hours for the maximum dose $(10 \mu \mathrm{M})$ tested (Figure $3(\mathrm{~b}))$. Interestingly, Dis as a single agent demonstrated significantly increased cytotoxicity against K12 compared with K7M2 at $640 \mathrm{nM}$. Dis treatment was also significantly more cytotoxic to $\mathrm{K} 12$ cells at the higher dose of $5 \mu \mathrm{M}$ (Figure 3(c)). Dis monotherapy did not reduce K7M2 viable cell signal beyond $50 \%$ from untreated controls up to $10 \mu \mathrm{M}$. An addition of an $800 \mathrm{nM}$ fixed $\mathrm{CuCl}_{2}$ supplement to Dis treatment resulted in clear potentiation in both $\mathrm{K} 12 \mathrm{IC}_{50}$ $60 \mathrm{nM}$ and K7M2 $\mathrm{IC}_{50} 120 \mathrm{nM}$ (Figure 3(f)). Further reduction to a $200 \mathrm{nM}$ fixed $\mathrm{CuCl}_{2}$ supplement also resulted in clear enhanced cytotoxic effects of Dis in both cell lines with K12 $\mathrm{IC}_{50} 50 \mathrm{nM}$ and K7M2 IC $5060 \mathrm{nM}$ (Figure 3(e)). Surprisingly, Dis supplemented with only $50 \mathrm{nM}$ of $\mathrm{CuCl}_{2}$ resulted in clear potentiation and reduction in effective cytotoxic dose to K12 cells only, with significant differences in \% live signal between OS cell lines from $80 \mathrm{nM}$ to $2.5 \mu \mathrm{M}$ (Figure 3(d)). With this very limited dose of $50 \mathrm{nM} \mathrm{CuCl} 2$ supplement, the Dis $\mathrm{IC}_{50}$ was $160 \mathrm{nM}$ for $\mathrm{K} 12$ and $4.4 \mu \mathrm{M}$ for K7M2, over a log difference in effective dose.

3.4. $\mathrm{CuCl}_{2}$-Potentiated Dis Requires Low-Dose Dox to Fully Eradicate K7M2 Cells In Vitro. To assess the long-term treatment effects on cytotoxicity and cellular recovery potential of highly metastatic OS more accurately, K7M2 was treated over 72 hours with monotherapies and combination treatments of Dox, Dis, and $\mathrm{CuCl}_{2}$. After viable cell counts were obtained, cells were monitored for recovery in fresh, treatment-free, culture media. K7M2 did not display any cytotoxicity over 72 hours at any $\mathrm{CuCl}_{2}$ doses tested 


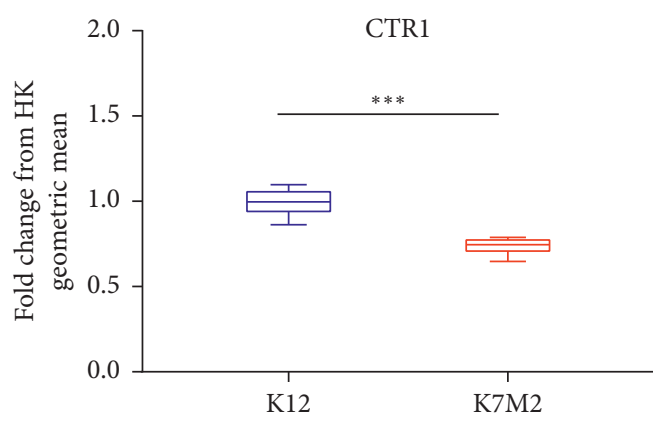

(a)

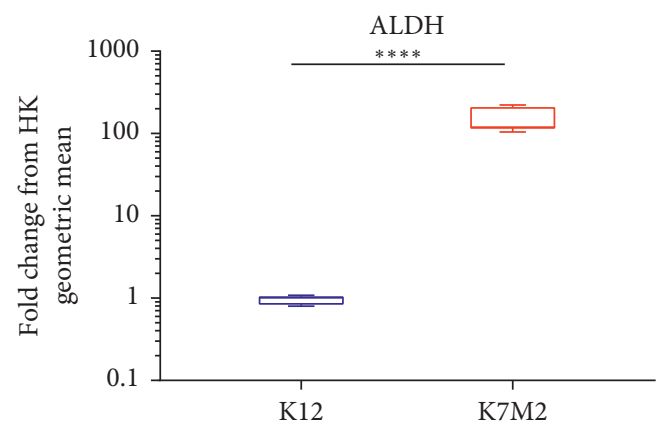

(c)

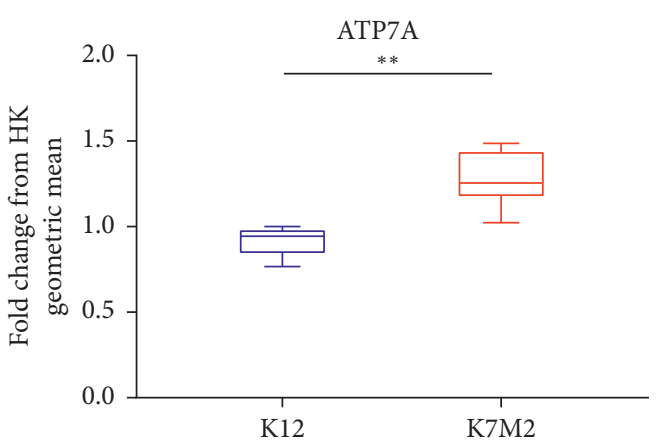

(b)

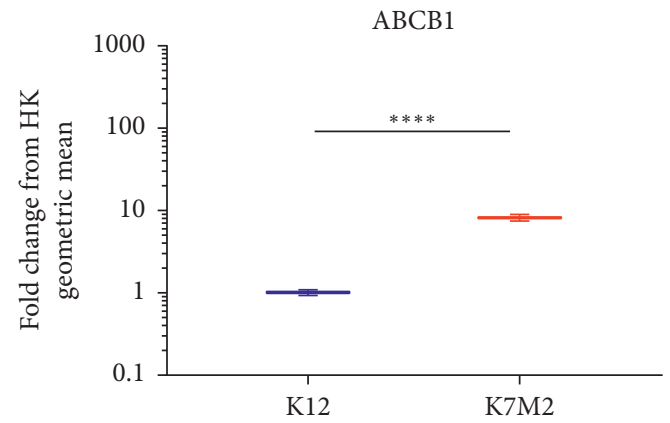

(d)

Figure 2: K7M2 displays altered expression of copper influx and efflux pumps as well as important chemoresistance factors compared to K12. K12 and K7M2 OS cell lines grown under identical culture conditions were processed for RNA extraction. cDNA generated was analyzed by qPCR for expression-level alterations between the OS cell lines and normalized to the geometric mean of housekeeper genes Nono, Rps17, and Rps18. (a) The Cu influx pump CTR1 was significantly downregulated in K7M2. (b) The Cu efflux pump ATP7A was significantly upregulated in K7M2 compared with K12. (c) Antioxidant enzyme and known cancer stem cell maker ALDH was significantly upregulated in K7M2 compared with K12 cells. (d) Additionally, the drug resistance multidrug pump ABCB1 was significantly upregulated in K7M2 compared with K12 cells. Molecular analysis was performed using technical triplicates from three separately cultured and processed samples $(n=9) .{ }^{* *} p<0.005 ;{ }^{* * *} p<0.0005 ;{ }^{* * * *} p<0.0001$.

$(0.12-2 \mu \mathrm{M})$ (Figure $4(\mathrm{a})$ ). High-dose Dis $(4 \mu \mathrm{M})$ reduced viable cell counts of K7M2 over $90 \%$ over 72 hours, but cells were able to grow in culture after drug removal. All $\mathrm{CuCl}_{2}-$ potentiated Dis combination doses tested resulted in $\sim 99 \%$ reduction of viable K7M2 cells, but remaining populations were able to grow in culture after drug removal. K7M2 cells treated with $600 \mathrm{nM}$ of doxorubicin displayed over $90 \%$ reduction in viable cells and were unable to grow in culture after drug removal (Figure 4(a)). We next treated K7M2 over 72 hours with sublethal doses of Dox $(100 \mathrm{nM})$, Dis $(100 \mathrm{nM})$, combination Dox and Dis (100 nM each), combination $\mathrm{Dis}+\mathrm{CuCl}_{2}(100 \mathrm{nM}$ and $250 \mathrm{nM}$ respectively), and triple treatment of all three drugs. Triple treatment of Dox, Dis, and $\mathrm{CuCl}_{2}(100 \mathrm{nM}, 100 \mathrm{nM}$, and $250 \mathrm{nM})$ resulted in $\sim 99 \%$ cell death after 72 hours of treatment, and, importantly, did not allow K7M2 cells to grow in culture after drug removal (Figure 4(b)).

\section{Discussion}

Here, we investigated endogenous $\mathrm{Cu}$ levels in murine OS cells with differing metastatic potentials and demonstrated that K12 cells had significantly greater endogenous intracellular $\mathrm{Cu}$ compared with the highly metastatic K7M2 cells. Evaluation of the expression levels for genes related to
$\mathrm{Cu}$ metabolism and drug resistance mechanisms also demonstrated altered levels of cellular $\mathrm{Cu}$ transporters between the two cell lines, which is associated with retention of $\mathrm{Cu}$ in $\mathrm{K} 12$ and $\mathrm{Cu}$ export in K7M2. Finally, we evaluated the ability of $\mathrm{CuCl}_{2}$ to affect the cytotoxicity of Dis and Dox and found that combination treatment could produce a durable, cytotoxic effect on the highly metastatic K7M2 cell line.

OS prognoses have not improved in several decades despite multiple clinical trials, forcefully illustrating that our current Dox-based strategies have reached the limits of their efficacy. Novel strategies directed at deliberately chosen biological targets are needed to improve prognoses for these patients. The use of Dis could have many possible uses in improving treatment success in metastatic OS. As a monotherapy, Dis could aid in chelating systemic copper found in the blood of patients, thereby attenuating the $\mathrm{Cu}$ associated metastatic mechanisms of OS. Dis could also be used as a chemosensitizer of metastatic OS cells, blocking ALDH antioxidant activity and improving the efficacy of ROS-inducing drugs like doxorubicin.

The ability of $\mathrm{CuCl}_{2}$ to augment the cytotoxic efficacy of Dis is well documented in the literature [30, 34-39]. There are multiple clinical trials in oncology using this combination [39-43], but not in sarcoma. We have demonstrated 


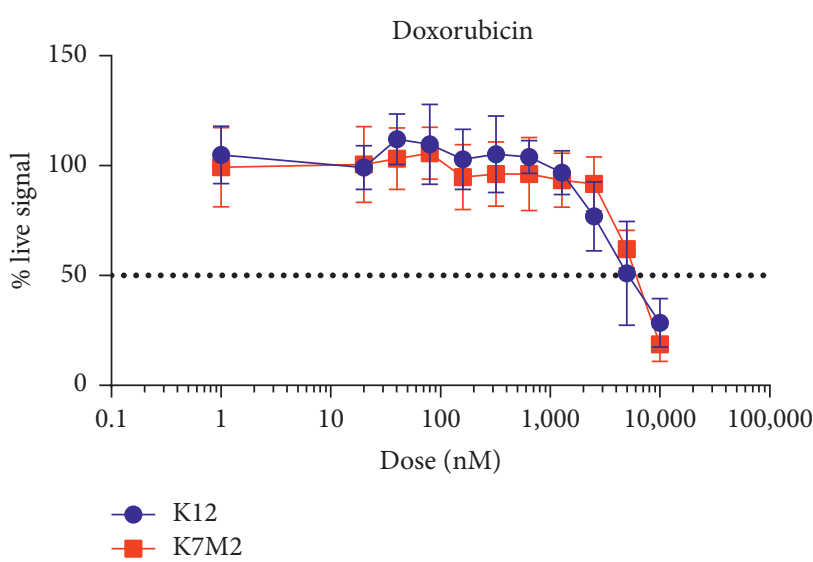

(a)

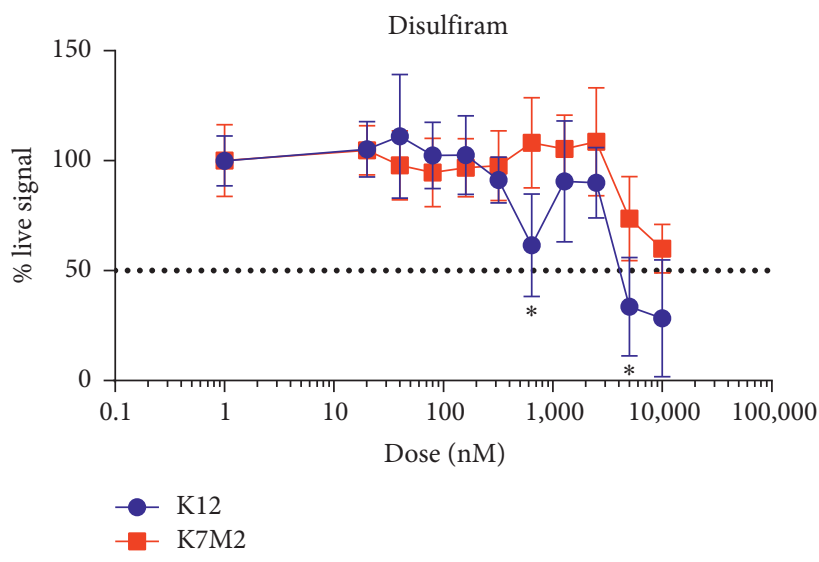

(c)

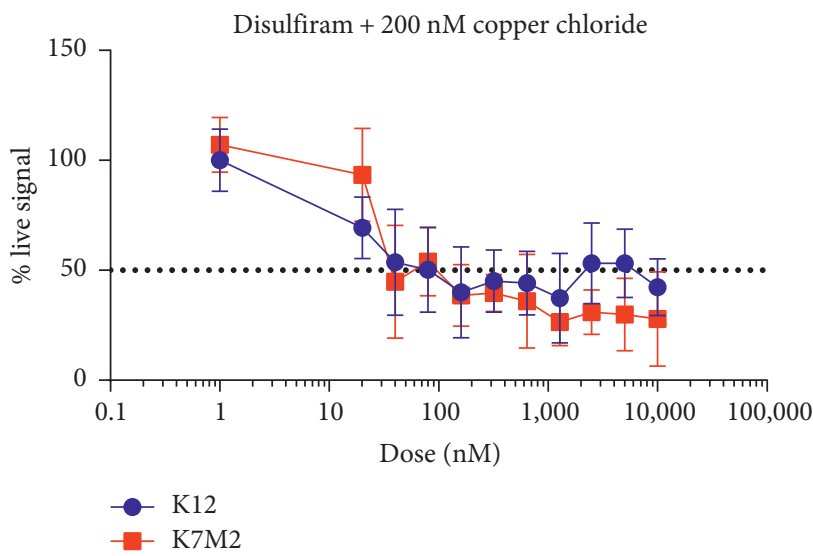

(e)

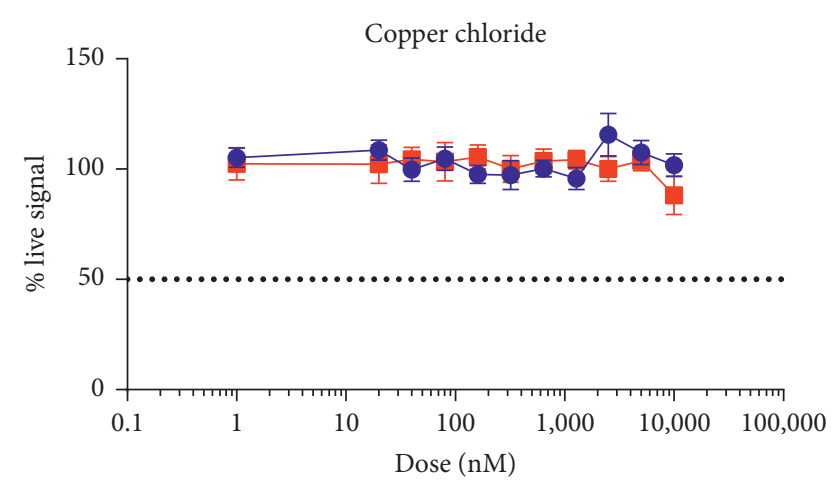

$\mathrm{K} 12$

K7M2

(b)

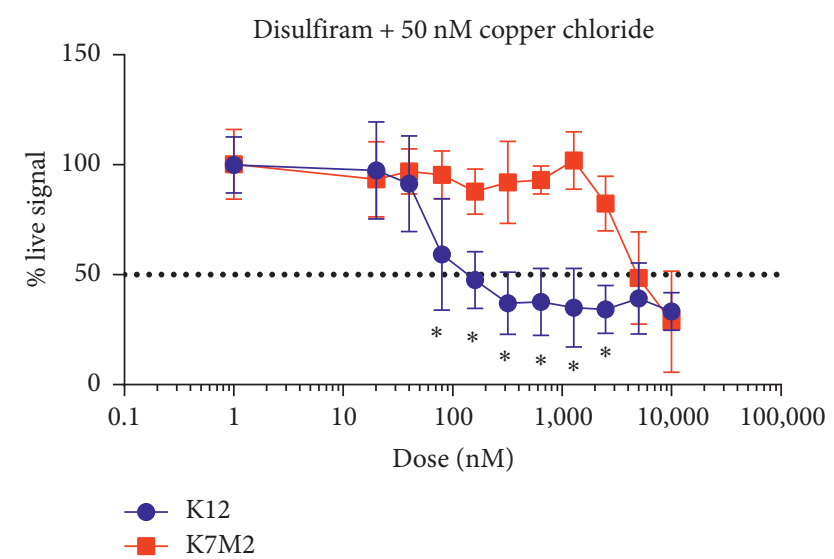

(d)

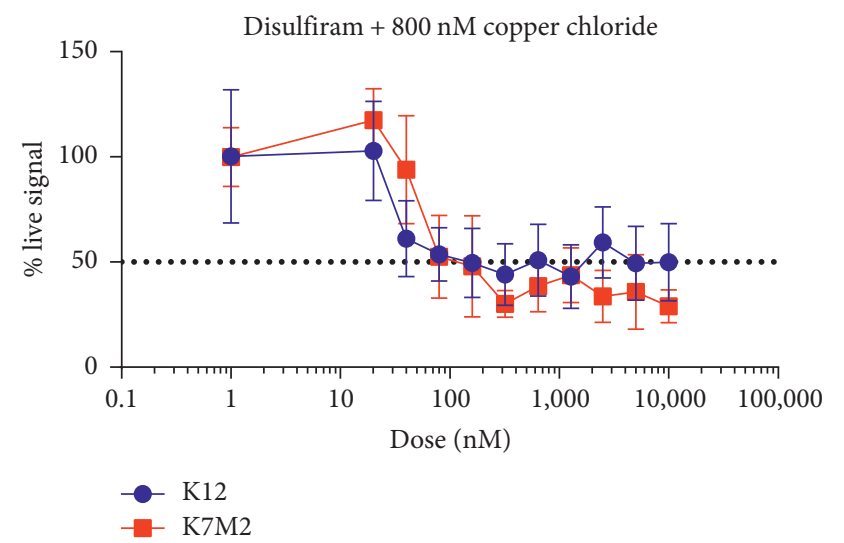

(f)

Figure 3: Copper potentiation of low-dose disulfiram selectively targets K12 cells compared to K7M2 cells. Both K12 and K7M2 cells were subjected to fold dilutions of Dox (a), $\mathrm{CuCl}_{2}$ (b), or Dis (c) single agent treatment over 24 hours. Additionally, cells were treated with fold dilutions of Dis $+\mathrm{CuCl}_{2}$ at fixed concentrations of $50 \mathrm{nM}$ (d), $200 \mathrm{nM}$ (e), or $800 \mathrm{nM}$ (f). After treatment, cells were stained with LIVE/ DEAD stain, and microscopy images were obtained. Live signal reduction from untreated controls was analyzed using FIJI image mean fluorescence measurements. $50 \mathrm{nM} \mathrm{CuCl}_{2}$ significantly decreased the live signal \% in $\mathrm{K} 12$ than in $\mathrm{K} 7 \mathrm{M} 2$ from $80 \mathrm{nM}$ to $2.5 \mu \mathrm{M}$ dose of Dis. All cytotoxicity experiments were performed in technical triplicate with three separate cell cultures $(n=9) .{ }^{*} p<0.05$.

that Dis has the capacity to affect the viability and metastatic phenotype of metastatic sarcoma cells in several publications [38], but neither we nor others have combined $\mathrm{CuCl}_{2}$ with Dis in sarcoma cells until now. The difference in susceptibility to Dis is likely related to differences in Cu metabolism, although the exact mechanism is a source of ongoing research for us and others.

Dox-based neoadjuvant and adjuvant chemotherapy is accepted worldwide as the standard of care in OS treatment and is felt to be responsible for the improvement in OS 


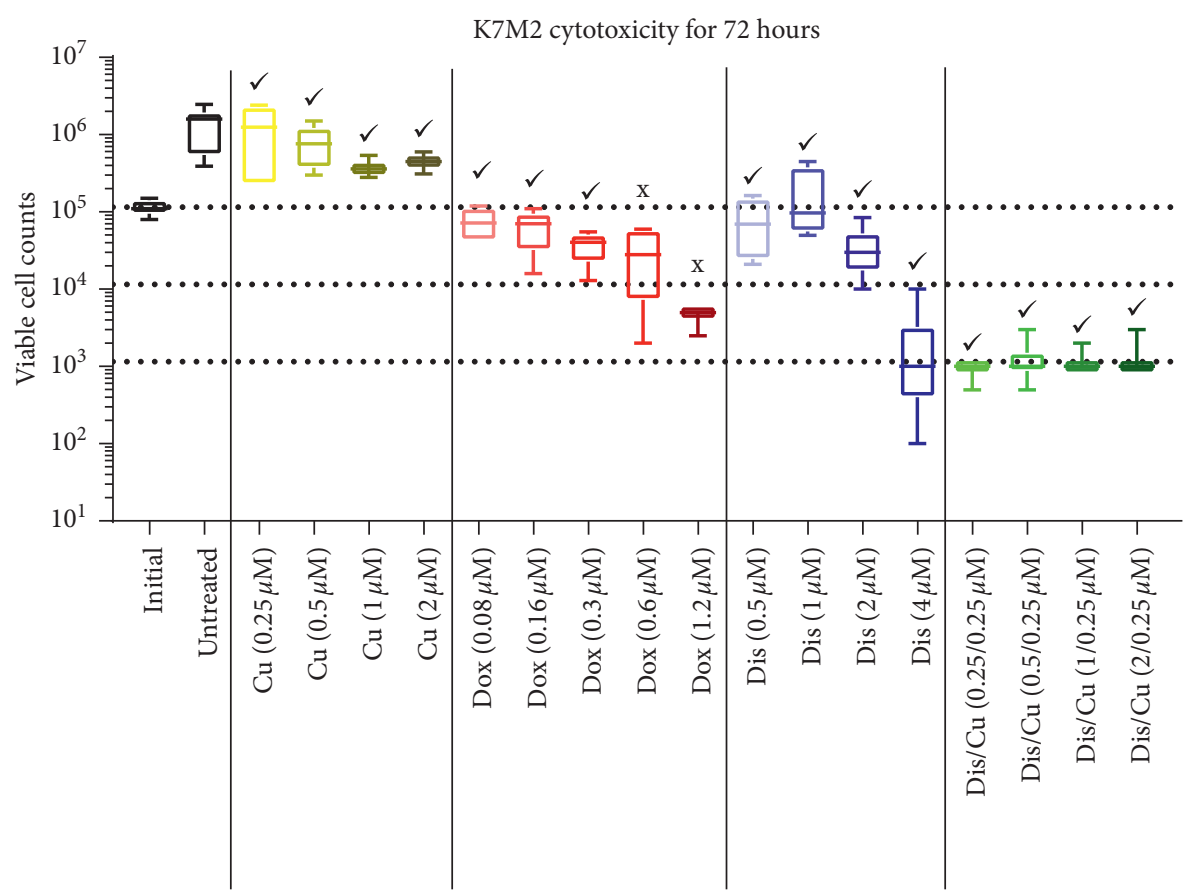

$\checkmark$ Cells could grow in culture after drug removal

$\mathrm{x}$ Cells could not grow in culture after drug removal

(a)

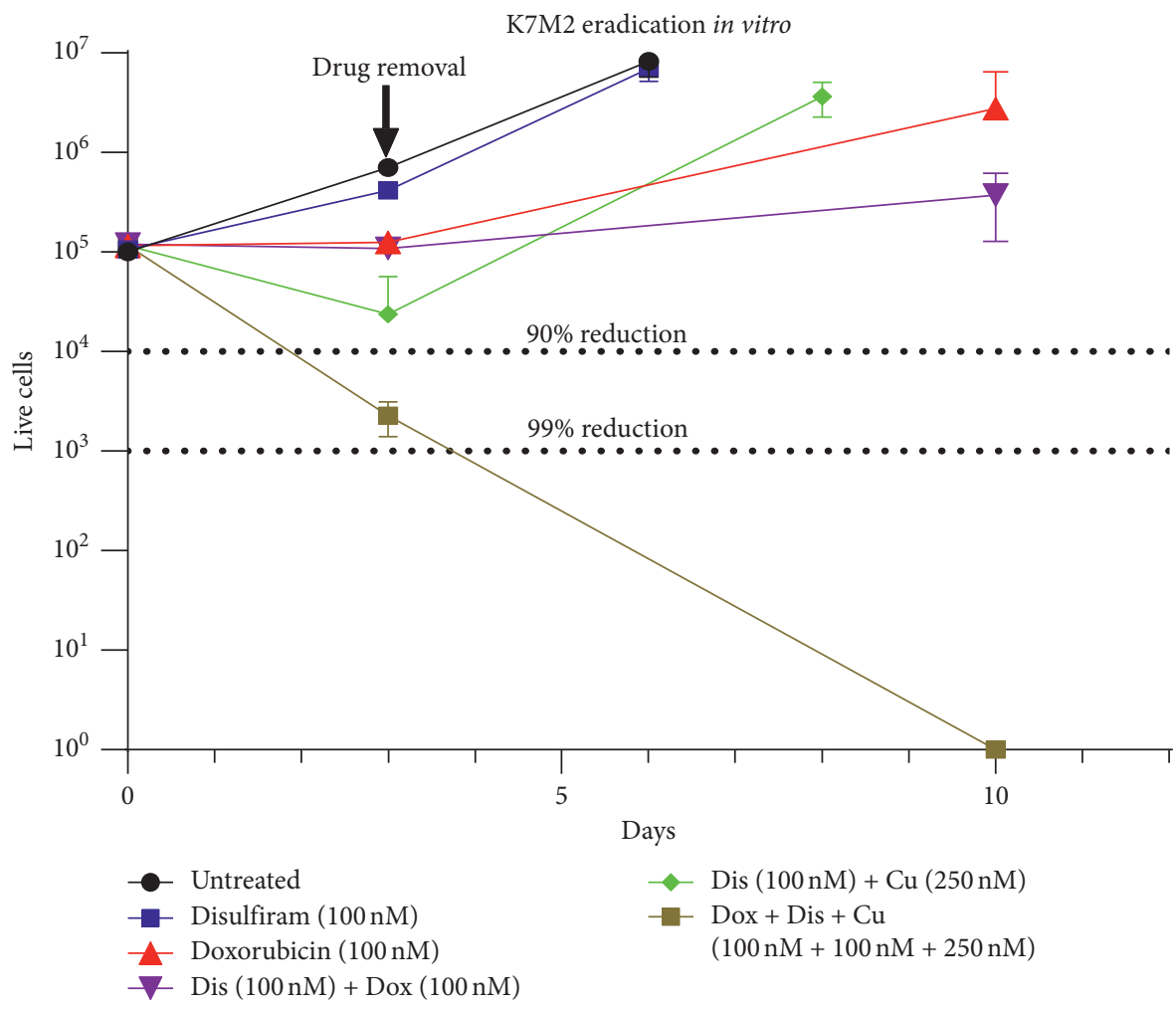

(b)

FIGURE 4: Combination treatment of doxorubicin, disulfiram, and copper chloride effectively reduces viability of K7M2 cells and eliminates recovery in vitro. (a) K7M2 OS cells were treated with $\mathrm{CuCl}_{2}(0.12-2 \mu \mathrm{M})$, Dox $(0.02-1.2,5 \mu \mathrm{M})$, Dis $(0.5-4 \mu \mathrm{M})$, and $\mathrm{Dis}+\mathrm{CuCl}_{2}(0.12$ and $0.25 \mu \mathrm{M}$ ) for 72 hours. After treatment, trypan blue exclusion staining was performed to obtain viable cell counts. Cells were cultured again in fresh media without drugs present and monitored for cellular growth. Dis potentiated with $\mathrm{CuCl}_{2}$ clearly killed the most cells over 72 hours, but all could subsequently recover in culture. (b) Dox and Dis combination treatment also allowed for cellular recovery in vitro, but triple treatment with Dox, Dis, and $\mathrm{CuCl}_{2}$ resulted in over $90 \%$ killing over treatment duration and treated cells did not recover after drug removal. Experiments were performed using three independent cell cultures $(n=3)$. 
survivorship compared with the prechemotherapeutic era $[3,14]$. It is therefore unlikely that oncologists will embrace novel treatment schemes that do not include Dox. This is clearly illustrated by the most recent OS clinical trials, which were designed to compare the standard of care versus the standard of care plus a novel agent $[3,14]$. With this reality in mind, we compared the ability of K7M2 cells to recover after treatment with Dox, Dis, $\mathrm{CuCl}_{2}$, or a combination of these. Only "triple treatment" with all three agents demonstrated cytotoxicity from which the cells did not recover. Interestingly, this effect was observed with low doses of Dox. This is clinically important because the cardiotoxic effects of Dox are known to be dose dependent. Any treatment strategy that decreases the cumulative dose of Dox would necessarily result in fewer cardiotoxicity complications.

There are several limitations to this study. First and most importantly, the experiments described above only used two murine OS cell lines for comparison. Although the K12 and K7M2 cell lines present a unique and powerful opportunity to compare related OS cells with differing metastatic phenotypes, it is still a small number of cell lines. We are presently seeking to rectify this deficiency by investigating these phenomena in established human OS cell populations, as well as primary cells from OS patients in our clinical practice with known metastatic histories.

\section{Conclusions}

In this study, we demonstrated differing levels of intracellular $\mathrm{Cu}$ in OS cell lines with different metastatic potentials. These differences are associated with the expression of genes related to $\mathrm{Cu}$ transport. Importantly, the addition of $\mathrm{CuCl}_{2}$ potentiates the cytotoxic effects of Dis. Finally, "triple treatment" with sublethal doses of Dox, Dis, and $\mathrm{CuCl}_{2}$ is the only experimental strategy we evaluated that leads to cell death from which cells could not recover. Based on these data, we theorize that highly metastatic and nonmetastatic OS cells utilize $\mathrm{Cu}$ differentially. We speculate that highly metastatic K7M2 cells actively pump $\mathrm{Cu}$ extracellularly to modulate the malignant processes of invasion and migration, possibly through matrix metalloproteinases (MMPs). Conversely, less metastatic K12 cells maintain higher amounts of intracellular $\mathrm{Cu}$. This would suggest that low levels of intratumoral $\mathrm{Cu}$ and high levels of serum $\mathrm{Cu}$ would portend a more aggressive and metastatic phenotype. We plan to verify this in experimental samples and patient-derived serum and tumors. We also have observed a relationship between Dox, Dis, and $\mathrm{CuCl}_{2}$ that should be evaluated in our preclinical mouse model of metastatic OS. Future studies will serve to determine how these powerful interactions might be exploited to the benefit of patients with metastatic OS.

\section{Data Availability}

The copper level, qPCR, and cell viability data used to support the findings of this study are available from the corresponding author upon request.

\section{Conflicts of Interest}

The authors declare no conflicts of interest.

\section{Acknowledgments}

Dr. Kurt Weiss was supported in part by the National Cancer Institute (K08CA177927-01 and R21CA199472) and Albert B. Ferguson, M.D., by the Orthopaedic Fund of the Pittsburgh Foundation. The authors are grateful for the support of Pittsburgh Cure Sarcoma and the Houy Family in loving memory of Jon Houy. This project used the Cancer Pharmacokinetics and Pharmacodynamics Facility (CPPF), UPMC Hillman Cancer Center, and was supported in part by award nos. P30CA047904 and R50CA211241. The authors thank Beth Stronach, Office of Research, for assistance.

\section{References}

[1] A. Luetke, P. A. Meyers, I. Lewis, and H. Juergens, "Osteosarcoma treatment-where do we stand? A state of the art review," Cancer Treatment Reviews, vol. 40, no. 4, pp. 523532, 2014.

[2] M. W. Bishop, K. A. Janeway, and R. Gorlick, "Future directions in the treatment of osteosarcoma," Current Opinion in Pediatrics, vol. 28, no. 1, pp. 26-33, 2016.

[3] J. S. Whelan, S. S. Bielack, N. Marina et al., "EURAMOS-1, an international randomised study for osteosarcoma: results from pre-randomisation treatment," Annals of Oncology, vol. 26, no. 2, pp. 407-414, 2015.

[4] S. S. Bielack, S. Hecker-Nolting, C. Blattmann, and L. Kager, "Advances in the management of osteosarcoma [version 1; referees: 2 approved]," F1000Research, vol. 5, p. 2767, 2016.

[5] S. S. Bielack, B. Kempf-Bielack, G. Delling et al., "Prognostic factors in high-grade osteosarcoma of the extremities or trunk: an analysis of 1,702 patients treated on neoadjuvant cooperative osteosarcoma study group protocols," Journal of Clinical Oncology, vol. 20, no. 3, pp. 776-790, 2002.

[6] G. Bacci, A. Briccoli, M. Mercuri et al., "Osteosarcoma of the extremities with synchronous lung metastases: long-term results in 44 patients treated with neoadjuvant chemotherapy," Journal of Chemotherapy, vol. 10, no. 1, pp. 69-76, 1998.

[7] G. Bacci, S. Ferrari, M. Mercuri et al., "Predictive factors for local recurrence in osteosarcoma: 540 patients with extremity tumors followed for minimum 2.5 years after neoadjuvant chemotherapy," Acta Orthopaedica Scandinavica, vol. 69, no. 3, pp. 230-236, 1998.

[8] G. Bacci, P. Picci, S. Ferrari et al., "Influence of adriamycin dose in the outcome of patients with osteosarcoma treated with multidrug neoadjuvant chemotherapy: results of two sequential studies," Journal of Chemotherapy, vol. 5, no. 4, pp. 237-246, 1993.

[9] P. A. Meyers and R. Gorlick, "Osteosarcoma," Pediatric Clinics of North America, vol. 44, no. 4, pp. 973-989, 1997.

[10] R. Mazanet and K. H. Antman, "Adjuvant therapy for sarcomas," Seminars in Oncology, vol. 18, no. 6, pp. 603-612, 1991.

[11] R. F. Williams, I. Fernandez-Pineda, and A. Gosain, "Pediatric sarcomas," Surgical Clinics of North America, vol. 96, no. 5, pp. 1107-1125, 2016.

[12] K. H. Antman, "Chemotherapy of advanced sarcomas of bone and soft tissue," Seminars in Oncology, vol. 19, no. 6, pp. 13-20, 1992. 
[13] P. Picci, "Osteosarcoma (osteogenic sarcoma)," Orphanet Journal of Rare Diseases, vol. 2, no. 1, p. 6, 2007.

[14] D. Carrle and S. S. Bielack, "Current strategies of chemotherapy in osteosarcoma," International Orthopaedics, vol. 30, no. 6, pp. 445-451, 2006.

[15] D. Reed and S. Altiok, "Metastatic soft tissue sarcoma chemotherapy: an opportunity for personalized medicine," Cancer Control, vol. 18, no. 3, pp. 188-195, 2011.

[16] J. Y. Blay, "Updating progress in sarcoma therapy with mTOR inhibitors," Annals of Oncology, vol. 22, no. 2, pp. 280-287, 2011.

[17] N. Penel, M. Van Glabbeke, S. Marreaud, M. Ouali, J. Y. Blay, and P. Hohenberger, "Testing new regimens in patients with advanced soft tissue sarcoma: analysis of publications from the last 10 years," Annals of Oncology, vol. 22, no. 6, pp. 1266-1272, 2011.

[18] E. Moussa, M. Zamzam, A. Kamel et al., "Risk stratification and pattern of cardiotoxicity in pediatric Ewing sarcoma," Journal of the Egyptian National Cancer Institute, vol. 29, no. 1, pp. 53-56, 2017.

[19] S. N. Hamilton, R. Carlson, H. Hasan, S. R. Rassekh, and K. Goddard, "Long-term outcomes and complications in pediatric ewing sarcoma," American Journal of Clinical Oncology, vol. 40, no. 4, pp. 423-428, 2015.

[20] C. Khanna, J. Prehn, C. Yeung, J. Caylor, M. Tsokos, and L. Helman, "An orthotopic model of murine osteosarcoma with clonally related variants differing in pulmonary metastatic potential," Clinical and Experimental Metastasis, vol. 18, no. 3, pp. 261-271, 2000.

[21] C. Khanna, J. Khan, P. Nguyen et al., "Metastasis-associated differences in gene expression in a murine model of osteosarcoma," Cancer Research, vol. 61, no. 9, pp. 3750-3759, 2001.

[22] K. R. Weiss, G. M. Cooper, J. A. Jadlowiec, R. L. McGough, and J. Huard, "VEGF and BMP expression in mouse osteosarcoma cells," Clinical Orthopaedics and Related Research, vol. 450, pp. 111-117, 2006.

[23] X. Mu, S. Patel, D. Mektepbayeva, A. Mahjoub, J. Huard, and K. Weiss, "Retinal targets ALDH positive cancer stem cell and alters the phenotype of highly metastatic osteosarcoma cells," Sarcoma, vol. 2015, Article ID 784954, 8 pages, 2015.

[24] X. Mu, C. Isaac, T. Schott, J. Huard, and K. Weiss, "Rapamycin inhibits ALDH activity, resistance to oxidative stress, and metastatic potential in murine osteosarcoma cells," Sarcoma, vol. 2013, Article ID 480713, 11 pages, 2013.

[25] X. Mu, C. Isaac, N. Greco, J. Huard, and K. Weiss, "Notch signaling is associated with ALDH activity and an aggressive metastatic phenotype in murine osteosarcoma cells," Frontiers in Oncology, vol. 3, p. 143, 2013.

[26] M. M. McManus, K. R. Weiss, and D. P. M. Hughes, "Understanding the role of Notch in osteosarcoma," Advances in Experimental Medicine and Biology, vol. 804, pp. 67-92, 2014.

[27] N. Greco, T. Schott, X. Mu et al., "ALDH activity correlates with metastatic potential in primary sarcomas of bone," Journal of Cancer Therapy, vol. 5, no. 4, pp. 331-338, 2014.

[28] D. Denoyer, S. Masaldan, S. La Fontaine, and M. A. Cater, "Targeting copper in cancer therapy: "Copper that Cancer"," Metallomics, vol. 7, no. 11, pp. 1459-1476, 2015.

[29] L. Fouani, S. V. Menezes, M. Paulson, D. R. Richardson, and Z. Kovacevic, "Metals and metastasis: exploiting the role of metals in cancer metastasis to develop novel anti-metastatic agents," Pharmacological Research, vol. 115, pp. 275-287, 2017.
[30] G. L. Fisher, V. S. Byers, M. Shifrine, and A. S. Levin, "Copper and zinc levels in serum from human patients with sarcomas," Cancer, vol. 37, no. 1, pp. 356-363, 1976.

[31] D. N. Breiter, R. B. Diasio, J. P. Neifeld, M. L. Roush, and S. A. Rosenberg, "Serum copper and zinc measurements in patients with osteogenic sarcoma," Cancer, vol. 42, no. 2, pp. 598-602, 1978.

[32] S. Tardito, I. Bassanetti, C. Bignardi et al., "Copper binding agents acting as copper ionophores lead to caspase inhibition and paraptotic cell death in human cancer cells," Journal of the American Chemical Society, vol. 133, no. 16, pp. 6235-6242, 2011.

[33] L. Duan, H. Shen, G. Zhao et al., "Inhibitory effect of Disulfiram/copper complex on non-small cell lung cancer cells," Biochemical and Biophysical Research Communications, vol. 446, no. 4, pp. 1010-1016, 2014.

[34] J. L. Allensworth, M. K. Evans, F. Bertucci et al., "Disulfiram (DSF) acts as a copper ionophore to induce copper-dependent oxidative stress and mediate anti-tumor efficacy in inflammatory breast cancer," Molecular Oncology, vol. 9, no. 6, pp. 1155-1168, 2015.

[35] X. Lun, J. C. Wells, N. Grinshtein et al., "Disulfiram when combined with copper enhances the therapeutic effects of temozolomide for the treatment of glioblastoma," Clinical Cancer Research, vol. 22, no. 15, pp. 3860-3875, 2016.

[36] D. J. Lewis, P. Deshmukh, A. A. Tedstone, F. Tuna, and P. O'Brien, "On the interaction of copper (II) with disulfiram," Chemical Communications, vol. 50, no. 87, pp. 13334-13337, 2014.

[37] P. E. Tawari, Z. Wang, M. Najlah et al., "The cytotoxic mechanisms of disulfiram and copper (II) in cancer cells," Toxicology Research, vol. 4, no. 6, pp. 1439-1442, 2015.

[38] J. A. Crasto, M. S. Fourman, A. Morales-Restrepo et al., "Disulfiram reduces metastatic osteosarcoma tumor burden in an immunocompetent Balb/c or-thotopic mouse model," Oncotarget, vol. 9, no. 53, pp. 30163-30172, 2018.

[39] View Clinical Trial NCT02963051-National Cancer Institute, https://www.cancer.gov/about-cancer/treatment/clinical-trials/ search/v?id=NCI-2017-00694\&r=1.

[40] Disulfiram in Treating Patients with Glioblastoma Multiforme after Radiation Therapy with Temozolomide-Full Text View-ClinicalTrials.gov, https://clinicaltrials.gov/ct2/show/ NCT01907165.

[41] Disulfiram and Gemcitabine Hydrochloride in Treating Patients with Unresectable Solid Tumors or Metastatic Pancreatic Cancer-Full Text View-ClinicalTrials.gov, https:// clinicaltrials.gov/ct2/show/NCT02671890.

[42] Disulfiram/Copper with Concurrent Radiation Therapy and Temozolomide in Patients with Newly Diagnosed Glioblastoma-Full Text View-ClinicalTrials.gov, https:// clinicaltrials.gov/ct2/show/NCT02715609.

[43] Disulfiram in Recurrent Glioblastoma-Full Text ViewClinicalTrials.gov, https://clinicaltrials.gov/ct2/show/NCT0 2678975. 


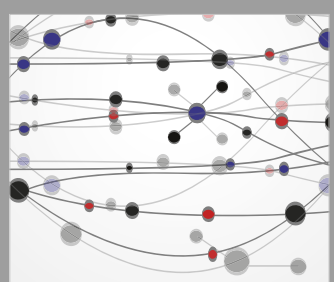

The Scientific World Journal
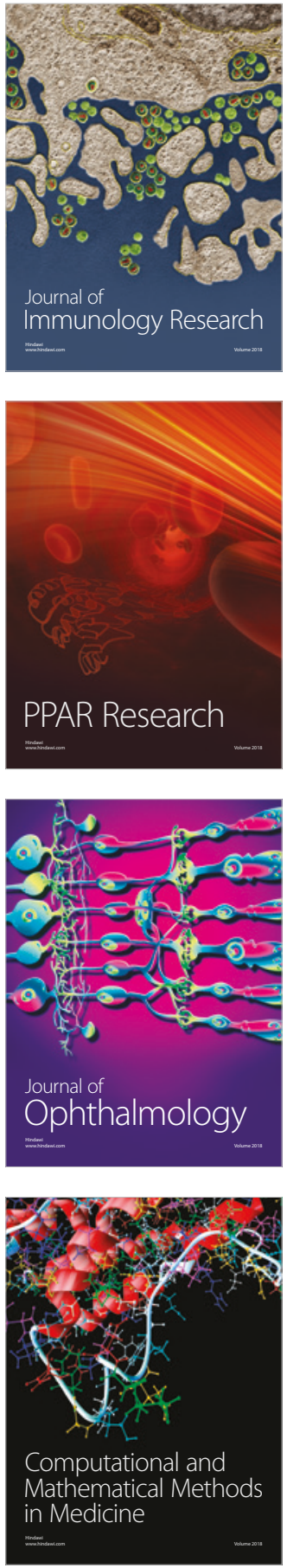

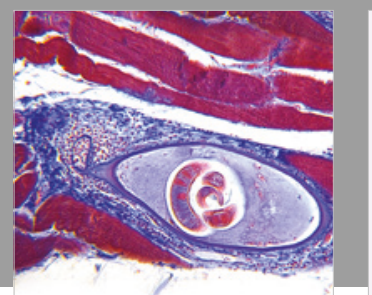

Gastroenterology Research and Practice

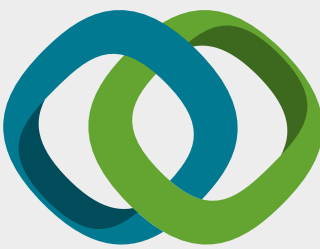

\section{Hindawi}

Submit your manuscripts at

www.hindawi.com
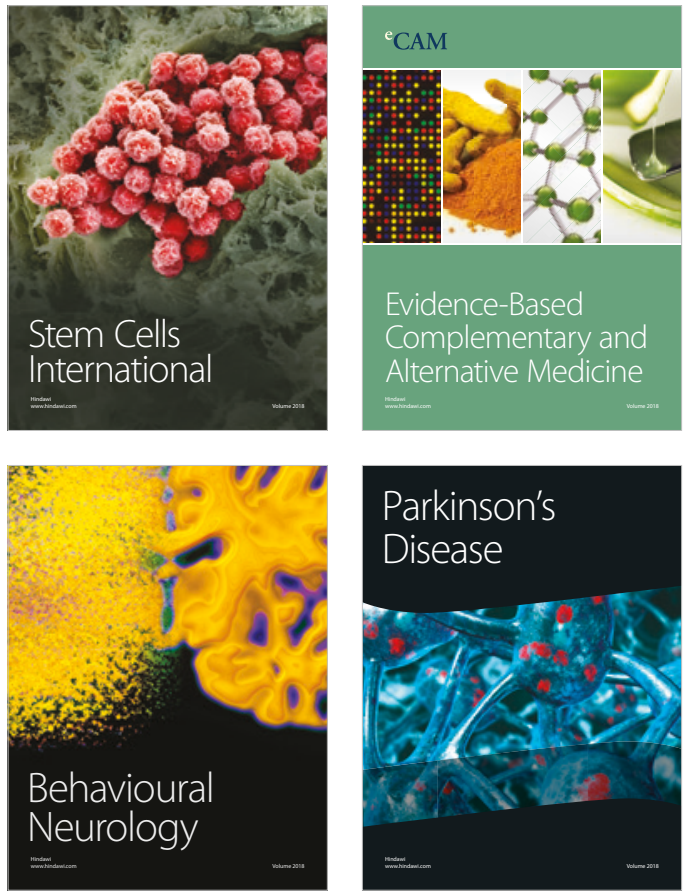

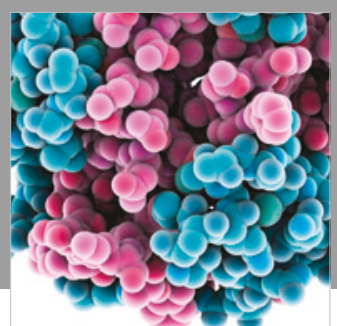

ournal of

Diabetes Research

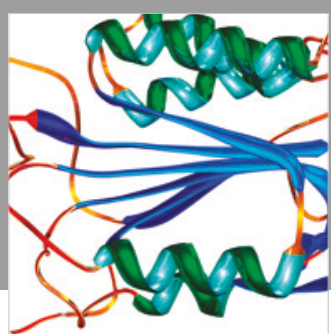

Disease Markers
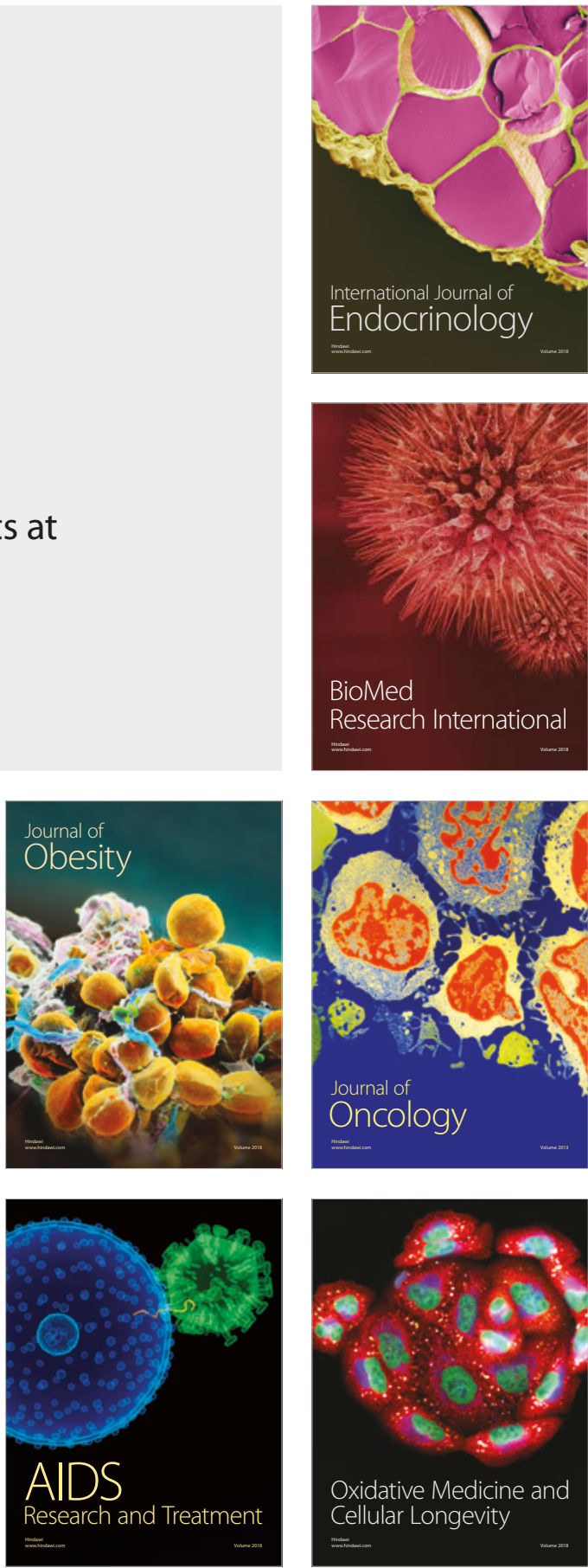